\title{
Der integrationistische Fehlschluss
}

Hauke Brunkhorst hat in der letzten Ausgabe des Leviathan den europäischen Hang zum technokratischen Regieren kritisiert und Auswege vorgeschlagen. ${ }^{1}$ In der EU, so der Autor, werden wichtige Entscheidungen zunehmend in einem depolitisierten Modus getroffen. Das kommt vor allem in der gegenwärtig zu beklagenden »Konstitutionalisierung der Austeritätspolitik « zum Ausdruck, womit der Fiskalpakt, das Verfahren zur Erkennung und Vermeidung makroökonomischer Ungleichgewichte und die Eingriffe der Troika in die Wirtschafts- und Sozialregime der Krisenländer Südeuropas gemeint sind. Zu Recht malt Brunkhorst die Demokratiewirkungen dieser Eingriffe in düsteren Farben. Seine Hoffnungen ruhen auf der Stück für Stück durchzusetzenden Politisierung und Demokratisierung der derzeit im technokratischen Modus getroffenen Entscheidungen. Die entsprechenden Zuständigkeiten, so Brunkhorst, müssen vom » institutionellen Gehäuse « aus EZB, Kommission und Rat in das Europäische Parlament (EP) verlagert und damit dem europäischen Parteienwettbewerb sowie der Deliberation einer europäischen Öffentlichkeit ausgesetzt werden. Das politische System der EU könne sich dann schrittweise zu einer parlamentarischen Demokratie im engeren Sinne entwickeln, in der europäische Parteien den Bürgerinnen und Bürgern alternative Politikentwürfe zur Auswahl stellen. Für die notwendige Aufwertung des EP und die ebenso notwendige Ausweitung der Zuständigkeiten der europäischen Gesetzgebung sieht Brunkhorst den Weg der Verfassungsinterpretation vor. Da entsprechende Vertragsänderungen nicht realisierbar erscheinen, setzt er auf die Bereitschaft und die Fähigkeit aller Beteiligten, die wünschenswerten Veränderungen nach und nach proaktiv in das europäische Primärrecht hineinzulesen. ${ }^{2}$

1 Brunkhorst 2014 a.

2 Unterschlagen wird hier, dass Brunkhorst seine Kritik am technokratischen Modus europäischen Regierens nicht nur auf die angesprochenen Überwachungs- und Korrekturverfahren bezieht, sondern auch auf die expansive Interpretation des europäischen Primärrechts durch den Europäischen Gerichtshof (EuGH), sofern sie Liberalisierung bewirkt (den Beitrag des EuGH zur politischen Konstitutionalisierung der EU begrüßt er hingegen nachdrücklich; siehe die Einzelheiten in Brunkhorst 2014 b). Ich stimme seiner Kritik ohne Einschränkung zu - vergleiche hierzu zusammenfassend Höpner 2014 - und teile seine Einschätzung, dass die vom EuGH verantworteten Grenzverschiebungen zwischen Staat und Markt in den Zuständigkeitsbereich der Politik gehören. Allerdings teile ich nicht die Auffassung, dass es sich bei den betreffenden Auseinandersetzungen im Wesentlichen um Links-Rechts-Konflikte handelt, sondern gehe davon aus, dass viele von ihnen Konflikte zwischen unterschiedlichen "Spielarten des Kapitalismus " sind (zu den Implikationen dieser Unterscheidung weiter unten). An dieser Stelle sei zudem auf eine fehlerhafte Interpretation des Keck-Urteils C-267/91 hingewiesen, das gerade keine Ausdehnung des Anwen- 
Die Brunkhorst'sche Argumentation steht - nicht in jedem Detail, aber in der Stoßrichtung - im Einklang mit zahlreichen aktuellen Beiträgen zur Integrationsdebatte, die ich nachfolgend unter dem Begriff "Integrationismus « zusammenfasse (siehe auch Scharpfs Antwort auf Habermas in diesem Heft). Das tiefergehende Problem, das ich in diesen Deutungen angelegt sehe, bezeichne ich als integrationistischen Fehlschluss. Gegenstand des Fehlschlusses ist die Überzeugung, jedes Problem der europäischen Integration lasse sich durch ein Noch mehr an Europa lösen, also durch eine über den Status quo hinausgehende Verlagerung von politischen Kompetenzen und demokratischen Prozessen auf das politische System der EU.

Dem integrationistischen Fehlschluss liegen drei Analysefehler zugrunde.

1. Ein Mangel an analytischer Zerlegung des Integrationsprozesses in Dimensionen der Integration, wobei insbesondere eine ökonomische, eine soziokulturelle und eine politische Dimension zu nennen wären (weitere, etwa eine judizielle, ließen sich hinzufügen). Erst diese analytische Zerlegung öffnet die Augen dafür, dass Integrationsschritte in einer Dimension (etwa: der wirtschaftlichen Integration) Desintegration in anderen Dimensionen (etwa: dem Zusammengehörigkeitsgefühl und Solidaritätsempfinden der Europäerinnen und Europäer, also der soziokulturellen Dimension) bewirken können. ${ }^{3}$ Nicht jedes Mehr an europäischer Regelsetzung führt dazu, dass die Europäerinnen und Europäer enger zusammenrücken und jenes "Wir «-Gefühl entwickeln, das wir uns als gute Europäer ${ }^{4}$ wünschen.

2. Ein Mangel an politökonomischer Fundierung. Jede Supranationalisierung politökonomischer Sachverhalte (etwa: die Währungsunion) muss hinsichtlich ihrer Eigenlogik untersucht werden, um die Wirkungen auf benachbarte Integrationsdimensionen erfassen zu können, und dabei zudem berücksichtigen, dass die EU ein in politökonomischer Hinsicht sehr heterogenes Gebilde ist, bestehend aus höchst unterschiedlichen Wohlstandsniveaus und - in der Terminologie der Vergleichenden Politischen Ökonomie - verschiedenen »Spielarten des Kapitalismus «. Die Unterschiedlichkeit nationaler Europäisierungsverläufe, entlang von Ländergrenzen verlaufende Konflikte über Geschwindigkeit und Richtung des Integrationsprozesses und persistente mitgliedstaatliche Integrationswiderstände lassen sich nur verstehen, wenn die politökonomische

dungsbereichs der europäischen Grundfreiheiten auf einen neuen Sachverhalt markierte, sondern die Ausnahme eines eng umrissenen regulativen Bereichs (Verkaufsmodalitäten) vom Anwendungsbereich der Warenverkehrsfreiheit. Interessant ist die Keck-Rechtsprechung im Licht aktueller Probleme, weil sich die Ausnahme bestimmter Regelungsfelder vom Anwendungsbereich der Grundfreiheiten im Prinzip nicht nur mit den Mitteln der Rechtsprechung, sondern auch politisch bewerkstelligen lassen sollte (ganz im Sinne Brunkhorsts), und weil eine solche Keck-Ausnahme eine stimmigere Antwort auf die Übergriffe des EuGH auf das mitgliedstaatliche Arbeitskampfrecht sein könnte als das vom Europäischen Gewerkschaftsbund (EGB) vorgeschlagene »soziale Fortschrittsprotokoll «.

3 Grundlegend: Eppler, Scheller 2013.

4 Gemeint sind hier und nachfolgend stets beiderlei Geschlechter. 
Heterogenität des zu integrierenden Gemeinwesens als Ausgangspunkt der Analyse ernst genommen wird. ${ }^{5}$

3. Ein Mangel an Analyse vorherrschender Konfliktlinien. Auch auf Ebene der EU werden Konflikte ausgetragen, die sich vor allem entlang unterschiedlicher Präferenzen von Klassen, Schichten und den ihnen nahestehenden Parteien und Organisationen sortieren. Die vorschnelle Annahme aber, die in den Mitgliedstaaten vorherrschende Konfliktlinie zwischen Links und Rechts dominiere auch auf EU-Ebene über alle weiteren "Nebenwidersprüche ", leitet zu fehlerhaften, ja kontraproduktiven Problemtherapien an. Die seit den Osterweiterungen noch einmal gewaltig gesteigerte Heterogenität der EU und die Schwere der gegenwärtigen Krise intensivieren Konflikte, die zwischen Ländergruppen verlaufen. Diese Lage der vorherrschenden Konfliktlinien macht einen entscheidenden Unterschied für die Demokratisierbarkeit der Konfliktaustragung und damit, mittelbar, für die Demokratisierbarkeit des politischen Systems der EU.

\section{Die Konvergenzerfordernisse fester Wechselkursregime und ihre Verletzung im Euro}

Die derzeitige Häufung neu eingeführter, aber auf Dauer gestellter technokratischer Politikmodi lässt sich nur unter Berücksichtigung der Funktionslogik des Euro verstehen. Im integrationistischen Mitte-Links-Spektrum wurde die Gründung der Währungsunion einhellig begrüßt - nicht nur weil die Währungsunion selbst einen historischen Integrationsschritt darstellte, sondern vor allem weil man hoffte, die gemeinsame Währung werde ihrerseits als Katalysator für die nachgelagerte Integration angrenzender Politikfelder wirken. Sowohl der inneren Funktionslogik der gemeinsamen Währung als auch der Heterogenität der ihr unterworfenen Wirtschafts- und Sozialregime wurde dabei wenig Beachtung geschenkt.

Der in unserem Zusammenhang entscheidende Punkt liegt in den Konvergenzerfordernissen fester Wechselkursregime. Auf zwei der vier üblicherweise zur makroökonomischen Anpassung zur Verfügung stehenden Instrumente mussten die EuroTeilnehmer unwiderruflich verzichten: auf die vor dem Hintergrund liberalisierter Kapitalmärkte ohnehin gering gewordenen (aber nicht gänzlich verschwundenen) Freiheitsgrade nationaler Geldpolitik und, wichtiger noch, auf die Wechselkurspolitik, die vormals als Korrektiv bei Auseinanderentwicklungen der Lohn- und Preisniveaus diente. Zählt man den Fiskalpakt als Bestandteil des Euro-Regimes mit, erfolgte mit dem Euro sogar der Verzicht auf drei der vier Instrumente makroökonomischer Anpassung. Aus diesem Grund potenzieren sich im Euro die Anforderungen an die Lohnpolitik und alle Politikfelder, die mittelbar auf den Lohn wirken: die Arbeitsmarktpolitik und die Sozialpolitik in all ihren Facetten. ${ }^{6}$ Soll die Währungsunion funktionieren, dann müssen sich die nominalen Lohnstückkosten der beteiligten Länder erstens dergestalt im Gleichklang entwickeln, dass von ihnen ein

5 Höpner, Schäfer 2015.

6 Scharpf 2011. 
mittelfristig gleichförmiger Inflationsdruck ausgeht. Und zweitens: Gehen von der gemeinsamen, sich an europäischen Durchschnittswerten orientierenden Geldpolitik dysfunktionale Impulse aus, lastet der Druck zur Gegensteuerung auf der Lohnpolitik und den lohnnahen Politikfeldern. Noch ungleich größer werden die Anforderungen, wenn sich - wie derzeit - die europäischen Preisniveaus bereits auseinanderentwickelt haben. Der gesamte Druck zur Korrektur der verzerrten effektiven Wechselkurse lastet dann auf der Lohn-, Arbeitsmarkt- und Sozialpolitik. $^{7}$

Angesichts der Heterogenität der nationalen Arrangements der Lohnfindung waren die auf die Lohnpolitik gerichteten Konvergenzerfordernisse aber von Anfang an unrealistisch. Der Norden mit Deutschland als Speerspitze betrieb Nominallohnzurückhaltung, im Süden hingegen stiegen die nominalen Lohnstückkosten stärker, als es mit dem 2-Prozent-Inflationsziel der EZB vereinbar sein konnte (nur Frankreich hielt sich ziemlich exakt an die Vorgaben). So darf dann auch nicht verwundern, dass es den nationalen Tarifsystemen derzeit nicht gelingt, aus sich heraus und ohne externe Eingriffe genau die Ergebnisse zu liefern, die zur Entzerrung der realen effektiven Wechselkurse notwendig wären. Weder wollte oder könnte irgendeine Gewerkschaft des Südens Deflationierung im erforderlichen Ausmaß einleiten, wenn sie als Organisation nicht untergehen will, noch sind die Sozialpartner des Nordens bereit, gezielt zu inflationieren, um den Süden von einem Teil des brutalen Deflationierungsdrucks zu entlasten. Vor diesem Hintergrund ist es nur konsequent, dass die EU mit dem Verfahren zur Erkennung und Vermeidung makroökonomischer Ungleichgewichte ein Instrument eingerichtet hat, das falsche, der Logik des Währungsregimes zuwiderlaufende Lohnpolitik mit Sanktionen belegt. ${ }^{8}$ Und ebenso konsequent ist, dass die Troika in den Krisenländern direkt in die Modi der Lohnfindung eingreift. ${ }^{9}$

Von der Lohnpolitik zur Arbeitsmarkt- und Sozialpolitik. Um die nominalen Lohnstückkosten zu senken, administriert die Troika den Krisenländern eine Rosskur aus Sozialabbau, Arbeitsmarktliberalisierung, Sparpolitik und gezielter Schwächung der Gewerkschaften. Wenn das denn notwendig ist, um die betroffenen Länder im Euro zu halten - warum betreiben die Regierungen der Krisenländer die erforderliche Austeritätspolitik nicht freiwillig? Weil sich Regierungsparteien gegenüber zwei Umwelten gleichzeitig verhalten müssen. Sie wollen einerseits Programme umsetzen und müssen sich andererseits so verhalten, dass sie wiedergewählt werden. Beides ist mit den Therapien zur Euro-Rettung unvereinbar. Keine Partei im Süden, und sei sie noch so neoliberal, vertritt ein Programm, das der verordneten Rosskur nur annähernd nahekommen könnte. Und radikaler Sozialabbau ist - aus Gründen, die insbesondere Paul Pierson herausgearbeitet hat - ein Selbstmordprogramm gegenüber den Wählern. ${ }^{10}$ Wie schon bei den Interventionen in die Tarifpolitik gilt

7 Flassbeck, Lapavitsas 2013; Hancké 2013; Höpner, Lutter 2014.

8 Ein exzessives Ungleichgewicht liegt demnach vor, wenn die nominalen Lohnstückkosten in drei aufeinanderfolgenden Jahren um jeweils mindestens drei Prozentpunkte steigen.

9 Siehe zu den Einzelheiten Schulten, Müller 2013.

10 Pierson 1996. 
auch hier: Es ist nur konsequent, dass die EU zur Krisenprophylaxe ein Verfahren sanktionsbewehrter Vorgaben installiert hat, das sich über die gesamte Bandbreite der Wirtschafts-, Arbeitsmarkt- und Sozialpolitik erstreckt, unabhängig von der primärrechtlich fixierten Kompetenzverteilung zwischen Mitgliedstaaten und EU. Und ebenso konsequent ist es im Sinne der Euro-Stabilität, dass die Troika direkt in die Wirtschafts- und Sozialsysteme der Krisenländer eingreift. Weder im Normalnoch im Krisenfall ist zu erwarten, dass die politischen Systeme der Euro-Teilnehmerländer jene Ergebnisse hervorbringen, die das feste Wechselkursregime erfordert.

\section{Die mangelnde Demokratisierbarkeit der Eingriffe}

Wenn die neuen europäischen, an die Mitgliedstaaten gerichteten Vorgaben also tatsächlich notwendig sind, um die Diskrepanz zwischen den Konvergenzerfordernissen des Euro einerseits und den tatsächlichen Operationen auf mitgliedstaatlicher Ebene andererseits zu verringern, lassen sie sich dann nicht zumindest mit mehr demokratischer Legitimation ausstatten? Um hier zu Klarheit zu gelangen, erscheint es hilfreich, die neuen Verfahren mit dem zu kontrastieren, was wir bis zur EuroKrise unter der »europäischen Politik « verstanden, deren Kern die Gemeinschaftsmethode war. Diese europäische Politik beruhte auf geteiltem Willen zur behutsamen Harmonisierung. Es wurden gemeinsame Standards vereinbart, die dann von allen teilnehmenden Ländern in nationales Recht überführt werden mussten. Inwieweit sich diese gemeinsame europäische Gesetzgebung weiter demokratisieren lässt, hängt vor allem von den gesellschaftlichen, vor-institutionellen Voraussetzungen transnationaler demokratischer Prozesse ab. ${ }^{11}$ Die gesteigerte Heterogenität der EU hat die Erfüllung dieser Voraussetzungen zwar nicht leichter gemacht. Aber es gibt keinen Grund, die Hoffnungen der Integrationisten vorschnell als Träumereien abzutun. Im Prinzip ist die europäische Gesetzgebung weiterer Demokratisierung zugänglich. Wenn sich aus den europäischen Parteienzusammenschlüssen Parteiorganisationen im engeren Sinne entwickeln, die den Wählern kohärente und voneinander unterscheidbare Programme zur weiteren Ausgestaltung der curopäischen Gesetzgebung zur Wahl stellen (bis heute sind wir hiervon weit entfernt), kann am Ende ein Mehr an Demokratie stehen.

Bei den neuen makroökonomischen Verfahren handelt es sich jedoch um etwas völlig anderes. Sie schaffen nicht gemeinsame Regeln, sondern formulieren an einzelne Länder gerichtete und nur diese Länder betreffende Vorgaben. Demokratie ist - so auch nachdrücklich Brunkhorst -, wenn Wahlen einen Unterschied machen. Wie aber sollten sich länderspezifische Vorgaben in unterscheidbare Programme bündeln lassen? Wie sollten europäische Parteien Programme zur Wahl stellen, die etwa besagen, dass Finnland und Dänemark eine etwas expansivere Fiskalpolitik fahren sollen, Italien und Frankreich hingegen eine stärker an Austerität orientierte,

11 Über Umfang und Erreichbarkeit dieser Voraussetzungen wird bekanntlich schon lange kontrovers diskutiert, etwa zwischen Dieter Grimm und Jürgen Habermas. 
dass Spanien seine Arbeitsmärkte und seine öffentliche Daseinsvorsorge liberalisieren, Griechenland Mindestlöhne senken und Belgien Kosten im Gesundheitswesen dämpfen soll? Keiner dieser Regelungsbereiche ließe sich aus sich heraus als demokratieresistent bezeichnen. Die Nicht-Demokratisierbarkeit der europäischen Eingriffe ergibt sich vielmehr aus der Gleichzeitigkeit und Verschränkung von erstens der Tatsache, dass es sich bei ihnen gerade nicht um Teilauswahlen größerer Möglichkeitsspielräume handelt, sondern um durchzusetzende Konvergenzerfordernisse, und zweitens der systematischen, unauflösbaren Inkongruenz von Urhebern einerseits und Betroffenen andererseits. Länderspezifische Vorgaben dieser Art sind nicht demokratisierbar.

Könnte man nicht zumindest das Europäische Parlament stärker einbinden? Die Vorstellung, für die länderspezifischen Vorgaben ließe sich mehr Legitimität dadurch gewinnen, dass man sie über die Schreibtische der Mitglieder von EP-Ausschüssen schickt, scheint mir zynisch. Spielräume demokratischer Auswahl zwischen politischen Alternativen entstünden hierdurch nicht. An den demokratiezerstörerischen Wirkungen der Eingriffe, auf die Brunkhorst zu Recht aufmerksam macht, würde sich daher nichts ändern. ${ }^{12}$ Den Parlamentariern des EP wäre zu raten, sich an den neuen Verfahren nicht die Hände schmutzig zu machen statt dafür zu streiten, ihnen Scheinlegitimation verleihen zu dürfen. ${ }^{13}$

\section{Links und Rechts auf europäischer Ebene}

Brunkhorst appelliert an die Europäer, die gemeinsame Strukturierung ihrer jeweiligen gesellschaftlichen Konflikte und ihrer Parteiensysteme durch die Links-RechtsAchse zu erkennen und den Gegensatz zwischen Links und Rechts auf europäischer Ebene zu re-konstitutionalisieren. ${ }^{14}$ " Grundlegende gesellschaftspolitische Alternativen müssen im ganzen Spektrum zwischen links und rechts wähl- und diskutierbar bleiben ", schreibt er zutreffend, "sonst gibt es keine Demokratie, sondern nur den falschen Schein «. ${ }^{15}$ Dass wir die Strukturierung politischer Konflikte entlang der sozioökonomischen, im Gegensatz von Kapital und Arbeit wurzelnden Konfliktachse in den meisten nationalen Demokratien als weiterhin dominant empfinden sie wird allerdings zunehmend von der quer zu ihr stehenden Konfliktachse libertär/

12 Es sei betont, dass dies nicht das Anliegen Brunkhorsts ist. Er hofft, die Eingriffe ließen sich durch ein Mehr an gemeinsamer europäischer Politik substituieren. Weder schwebt ihm - zu Recht, wie ich meine - eine bessere Legitimitätszufuhr durch mehr Einbindung des EP noch eine sozialere Ausrichtung der neuen Korrekturverfahren durch Hinzufügung sozialer Beurteilungskriterien vor (beide Ideen finden sich in den Forderungskatalogen der Parteien, Organisationen und Stiftungen des Mitte-Links-Spektrums).

13 Ähnlich jüngst auch Watkins 2014, S. 20-23.

14 In Das doppelte Gesicht Europas akzentuiert Brunkhorst diesen Argumentationsschritt etwas anders: Er betont, ganz im Sinne meines Einwands, den »Kalten Krieg zwischen Nord- und Südeuropa «, hält aber eine "Umpolung " des Konflikts in einen transnationalen Klassenkampf für möglich (siehe die Einzelheiten in Brunkhorst 2014 b, Kapitel VI).

15 Brunkhorst 2014 a, S. 519. 
autoritär durchbrochen -, ${ }^{16}$ bedeutet aber noch nicht, dass diese Achse auch die Konflikte auf supranationaler Ebene strukturiert. Angesichts der immer heterogener gewordenen EU und der in ihr seit dem Ausbruch der Euro-Krise geradezu explodierten Verteilungskonflikte verlaufen die entscheidenden öffentlichen europäischen Auseinandersetzungen derzeit eher zwischen Ländergruppen als entlang einer transnationalen Links-Rechts-Achse. ${ }^{17}$

Die Implikation dieser Unterscheidung sei anhand eines Beispiels verdeutlicht. In allen Mitgliedstaaten sorgten die Kommissionspläne für eine europäische Frauenquote für politische Auseinandersetzungen, und in allen Ländern gab es eher progressive Kräfte, die die Kommission zu mutigem und forschem Vorgehen ermunterten, und eher konservative Kräfte, die vor zu weitgehenden Eingriffen warnten. Wäre dieser Konflikttyp für die Mehrzahl der auf europäischer Ebene ausgetragenen Konflikte repräsentativ - warum sollten die Hoffnungen der Integrationisten dann nicht aufgehen können, wenn auch gewiss nicht in einem big bang, ${ }^{18}$ sondern in einem sich behutsam vollziehenden historischen Prozess? Warum sollte es den europäischen Parteien nicht gelingen, kulturelle Hürden zunehmend zu überwinden und die nationalen Links-Rechts-Auseinandersetzungen auf europäischer Ebene zu reaggregieren? Die Finalität dieses Prozesses könnte dann tatsächlich ein europäisches Parteiensystem sein, das idealerweise in einem politischen System mit einem mit Initiativrecht ausgestatteten Parlament und Wahlen mit gleichem Stimmengewicht ${ }^{19}$ operieren würde.

Wie repräsentativ aber ist das Beispiel der europäischen Frauenquote? Gewagt war die Annahme einer Dominanz der transnationalen Konfliktachse zwischen Links und Rechts schon immer. Denn wann immer die europäische Politik asymmetrische institutionelle Anpassungslasten oder deutliche transnationale Verteilungswirkungen hervorzubringen drohte, verlief die dominante Konfliktlinie eher zwischen Ländern als zwischen Parteien. ${ }^{20}$ Soll es den Kapitalmarktteilnehmern erlaubt sein, die Führungskräfte von Unternehmen mit der glaubhaften Drohung feindlicher Übernahmen zu disziplinieren, und wenn ja, mit welchem Nachdruck? Wie könnte ein Modell der Arbeitsnehmermitbestimmung auf Ebene der Leitungsorgane von Unternehmen aussehen, mit dem alle Länder leben können (sollte es so

16 Kitschelt 1988. Die Hoffnungen der Integrationisten hebt die Existenz dieser »Querachse " freilich nicht aus den Angeln, denn auch der Konflikt zwischen Libertären und Autoritären ließe sich im Prinzip auf supranationaler Ebene abbilden und austragen.

17 Sehr anschaulich zum Konflikt zwischen Gläubigern und Schuldnern Blomert 2012 sowie zu fiskalischen Verteilungskonflikten Streeck, Elsässer 2014.

18 Vergleiche hierzu den von Ulrich Beck initiierten und unter anderem von Jürgen Habermas unterschriebenen Aufruf zur letzten Europawahl, der die Benennung von Spitzenkandidaten der europäischen Parteienfamilien als demokratischen »Quantensprung " bewertete und der sich hier findet: http://waehlt-europa.de/ (Zugriff vom 06.01.2015).

19 Gerade angesichts des Umfangs an Konflikten, die Brunkhorst gern im EP behandelt sähe, stutze ich über seine Geringschätzung der seinem Eindruck nach weniger wichtigen, weil "ohnehin nirgends verwirklichte[n] regulativen Idee, jeder Nase eine Stimme zu gewährleisten «; Brunkhorst 2014 a, S. 516.

20 Grundlegend: Scharpf 1999, Abschnitt 2.3.3. 
etwas überhaupt geben)? Wie viel schwächeres Arbeitsrecht dürfen transnational entsandte Arbeitnehmer in die stärker regulierten Hochlohnökonomien einführen? Bei all diesen Konflikten standen sich schon vor Ausbruch der Euro-Krise zuvörderst Ländergruppen mit unterschiedlichen Vorstellungen gegenüber. ${ }^{21}$

Seit der Euro-Krise indes ist die Annahme einer Dominanz der transnationalen Links-Rechts-Konfliktachse nicht nur gewagt, sondern gänzlich irreführend. Die Euro-Krise hat eine tiefe Spaltung entlang der Nord-Süd-Achse bewirkt, die Länder mit Leistungsbilanzdefiziten und -überschüssen, Gläubiger und Schuldner sowie Befürworter und Gegner strikter austeritätspolitischer Vorgaben voneinander trennt. Ob man nun an die Ver- oder Entschärfung der neuen Überwachungsverfahren denkt, an die unkonventionelle Geldpolitik der EZB, an die Diskussion über Euro-Bonds oder einen etwaig zu errichtenden europäischen Finanzausgleich - stets sind es gebündelte Länderinteressen, die in den Auseinandersetzungen aufeinanderprallen, während sich die Positionen der großen Parteien innerhalb der Länder allenfalls marginal unterscheiden, wenn überhaupt. ${ }^{22}$ Die deutschen Volksparteien CDU/CSU und SPD bieten hervorragendes Anschauungsmaterial. Dekliniert man die genannten europäischen Auseinandersetzungen anhand der Europawahlprogramme der großen inländischen Volksparteien durch, dann ist schwer zu erkennen, an welchen Stellen sich die europapolitischen Vorstellungen der Parteien eigentlich unterscheiden.

Für das politische System Europas hat die - heute geradezu erdrückende - Dominanz der länderbezogenen Konfliktachse zwei Konsequenzen. Erstens, demokratische Steuerung der Politik über Wahlen läuft leer. Gewählt werden Parteien, nicht Länder. Wie viele Abgeordnete Finnland oder Spanien in das EP schickt, steht bereits vor dem Wahlakt fest - Wahlen machen also keinen Unterschied. Und zweitens, werden diese Konflikte gleichwohl politisiert, können sie kaum etwas anderes bewirken als die Verfestigung nationaler Ressentiments, also soziokulturelle Desintegration. Vor dieser Gefahr hat Fritz W. Scharpf nachdrücklich gewarnt. ${ }^{23}$ Niemand muss daran erinnert werden, welche nationalen Stereotypen in den Auseinandersetzungen über die Verteilung der Anpassungslasten der Krisenbewältigung freigesetzt wurden, sobald die Konflikte auf die Straßen getragen, sprich: politisiert wurden. Die von Brunkhorst eingeforderte Politisierung droht in solchen Konstellationen offenbar sogar das Gegenteil dessen zu bewirken, wofür sie eigentlich antritt. An dieser Stelle kann der integrationistische Fehlschluss, so gut der ihn antreibende Zweckoptimismus auch gemeint sein mag, erheblichen Schaden anrich-

21 Angesprochen sind hier die Auseinandersetzungen um die Übernahmerichtlinie, die SERichtlinie, die Entsenderichtlinie und die Dienstleistungsrichtlinie.

$22 \mathrm{Zu}$ diesem Ergebnis kommt auch Aleksandra Maatsch bei ihrer Analyse der politischen Konflikte im Zuge der Errichtung der neuen Institutionen und Regeln europäischer economic governance (siehe Maatsch 2014 sowie ihre aktuellen Forschungen).

23 Scharpf 2015. 
ten. ${ }^{24}$ Die europäischen Parteienfamilien haben dieses Problem zweifellos erkannt. Eben deshalb wurden wir im Mai 2014 Zeugen eines Gespensterwahlkampfs, in dessen Rahmen die Parteien die (ja derzeit nicht gerade knappen) schweren Konflikte der europäischen Politik konsequent umschifften. ${ }^{25}$

\section{Der integrationistische Fehlschluss am Beispiel des europäischen Mindestlohns}

Ich schließe meine Argumentation mit einer Kritik an Brunkhorsts an die Gewerkschaften gerichteten Vorschlägen, in denen nahezu alle oben diskutierten Bestandteile des integrationistischen Fehlschlusses wie auf einer Perlenkette aufgereiht scheinen. Das demokratische Europa, so der Autor, erfordere eine "Transnationalisierung des Klassenkampfs « ${ }^{26}$ Um diese voranzubringen, schlägt er die Forderung nach einer einheitlichen - explizit nicht: relativen, sondern: einheitlichen - europäischen Lohnuntergrenze vor. "Fehlen nur noch die Gewerkschaften ", schreibt Brunkhorst, »die bereit wären, dafür transnationale Streiks zu organisieren «.27 Betrachten wir die Voraussetzungen und Implikationen dieser Idee eingehender.

Zunächst einmal unterschätzt der Appell die Spannweite der in der EU vertretenen Produktivitätsniveaus. Ein Blick auf die derzeit wirksamen nationalen Mindestlöhne illustriert diese Unterschiede. Der niedrigste gesetzliche Mindestlohn findet sich derzeit mit etwa einem Euro pro Stunde in Bulgarien, der höchste mit etwas über elf Euro in Luxemburg. ${ }^{28}$ Jeder Mindestlohn, der für Beschäftigte im Norden noch irgendeinen Unterschied machen würde, müsste in den Volkswirtschaften des Ostens gewaltige Schäden anrichten. Und jeder Mindestlohn, der auf die östlichen Länder passen würde (dort könnten die Mindestlöhne gewiss etwas höher sein als derzeit), würde für die Beschäftigten des Nordens keinen Unterschied machen. Ein

24 Dieser Punkt ist meines Erachtens wichtig für die europawissenschaftliche »No demos? «-Debatte (vgl. jüngst Risse 2014). Mit der horizontalen Europäisierung (Berichterstattung über andere europäische Länder), der vertikalen Europäisierung (Berichterstattung über die Vorgänge in den europäischen Institutionen und Organisationen) und der europäischen Politisierung (kontroverse Debatten über europäische Probleme) können drei wichtige Voraussetzungen der Entstehung einer europäischen Öffentlichkeit erfüllt sein - und den Status quo gleichwohl von den vor-konstitutionellen Voraussetzungen einer europäischen Demokratie weiter entfernen.

So erscheint mir dann auch der bei Gerhards und Lengfeld (auf die Brunkhorst verweist) erfolgende Schluss von der abstrakten Zustimmung der Bürger zu europäisch geschützten Individualrechten (oder etwa zur Forderung nach einer Tobin-Steuer) auf » einen erstaunlich hohen Grad der [europäischen] Sozialintegration " (Gerhards, Lengfeld 2013, S. 213), auf die Konstitution eines "europäischen Volks (ebd., S. 216) und die Erfüllung der sozialen Voraussetzungen für europäische Demokratie und Umverteilung (ebd., S. 215 und 222) mutig.

25 Was dann freilich auch ein zweifelhaftes Licht auf die These vom demokratischen Quantensprung wirft (vgl. Fußnote 18).

26 Ausführlich: Brunkhorst 2014 b, Kapitel VI.

27 Ebd., S. 517.

28 Daten: Schulten 2014, S. 7. 
zwingenderes Beispiel für ein Regelungsfeld, in dem einheitliche europäische Vorgaben nicht wünschenswert sein können, ist daher kaum denkbar. Eine europäische Mindestlohnregelung könnte überhaupt nur eine relative sein (etwa: ein vom nationalen Medianlohn abgeleiteter Korridor, in dem sich die nationalen Mindestlöhne bewegen dürfen). ${ }^{29}$

Aber auch die Forderung nach einem relativen europäischen Mindestlohn ignoriert die Heterogenität der beteiligten Länder - genauer: die Verschiedenheit der nationalen Regime der Lohnfindung -, und der Kampf für sie würde daher gerade nicht die "Transnationalisierung des Klassenkampfs « bewirken, die sich Brunkhorst erhofft. Denn für die nordeuropäischen Gewerkschaften wären europäische Mindestlohnvorgaben gerade keine Siege im Klassenkampf, sondern eine Beschädigung ihrer Machtressourcen, die sie unter anderem in konsequenter staatlicher Nichteinmischung in alle Lohnfragen erkennen. Diese Unterschiedlichkeit nationaler Traditionen muss ernst genommen und respektiert werden. ${ }^{30}$ Man stelle sich eine Kampfabstimmung in der europäischen Sozialdemokratie oder im Europäischen Gewerkschaftsbund (EGB) über Brunkhorsts Idee vor. Sie würde nichts anderes bewirken, als die schwedische Linke (noch) weiter vom europäischen Projekt zu entfernen. Jeder Versuch, den nordeuropäischen Mitgliedsländern Mindestlöhne aufzuzwingen, würde keine Europäisierung politischer Auseinandersetzungen bewirken, sondern antieuropäische Ressentiments stärken und die EU von den vorinstitutionellen Voraussetzungen einer transnationalen Demokratie damit weiter entfernen. Und gänzlich abwegig ist die Vorstellung, man könne schwedische Gewerkschafter zur Beteiligung an entsprechenden transnationalen Streiks bewegen. Streiken würden sie allenfalls gegen europäische Einmischungen in ihr Tarifmodell. ${ }^{31}$ Am Rande sei erwähnt, dass ähnliche Vorbehalte gegen europäische Mindestlohnvorgaben auch unter den Gewerkschaften Österreichs und - wohl abgeschwächt - Italiens existieren. ${ }^{32}$

Und selbst wenn wir uns all das wegdenken - ein größeres Eigentor als die Forderung nach einer europäischen Mindestlohnrichtlinie könnten die europäischen

29 Unklar bleibt gleichwohl, warum Brunkhorst (im Anschluss an Gerhards, Lengfeld 2013, S. 217) meint, ein einheitlicher, für Deutschland also mutmaßlich viel zu niedriger europäischer Mindestlohn werde Lohneinbußen in Deutschland bewirken - es soll sich doch um eine Lohnuntergrenze handeln und nicht um eine Lohnobergrenze?

30 Den Mangel an Respekt vor den schützenswerten Beständen nationaler Autonomie halte ich für den schwerwiegensten Denkfehler - eher eine falsche Prämisse als ein Analyseschritt - der Integrationisten. Leider kann ich auch bei Brunkhorst keine Beachtung mitgliedstaatlicher Institutionen entdecken, bei denen Bewahrung vor europäischen Übergriffen legitim erschiene. Auf den Punkt gebracht: "Der nationale Staat muss, so könnte man mit Hegel sagen, untergehen, um aufgehoben zu werden « (Brunkhorst $2014 \mathrm{~b}$, S. 165). Diese Radikalität - die nicht nur bei Brunkhorst zu finden ist - macht ratlos: Sehen die Integrationisten nicht, dass ihr Programm gerade wegen dieser Geringschätzung legitimer nationaler Eigenheiten zum Scheitern verurteilt ist?

31 Selbst ein Austritt der schwedischen Gewerkschaften aus dem Europäischen Gewerkschaftsbund erschiene in so einem Fall nicht ausgeschlossen.

32 Ich danke Martin Seeliger für diese Hinweise. 
Gewerkschaften derzeit meiner Einschätzung nach kaum schießen. Denn gegenwärtig schließt Art.153 Abs.5 AEUV europäische Richtlinien zu Lohn- und Arbeitskampffragen kategorisch aus. Es müsste also zunächst einmal eine Kompetenznorm installiert werden. Sollten die Gewerkschaften eine solche Kompetenzgrundlage fordern (einmal abgesehen davon, dass zurzeit keine Vertragsrevision in Sicht ist)? Ich hielte das für einen schweren strategischen Fehler. Denn die Stellungnahmen der Kommission zu Viking und Laval, ihr derzeitiger Umgang mit den Lohnregimen der Krisenländer und die gescheiterte Monti II-Verordnung zur Errichtung eines Erfassungsregimes für Arbeitskämpfe zeigen unmissverständlich, was sie mit einer Befugnis zur Vorlage von entsprechenden Richtlinien- und Verordnungsvorschlägen anfangen würde. Ohne die Sperrklausel des Art. 153 Abs. 5 AEUV hätten die zahlreichen Subsidiaritätsvorbehalte gegen Monti II nicht zum Erfolg geführt. ${ }^{33}$ Immer wieder verdeutlicht die Kommission, dass sie oberhalb der Unternehmensebene angesiedelte Lohnfindung für ein Wettbewerbshindernis hält und Arbeitskämpfe mit transnationaler Wirkung für Binnenmarktstörungen. Solange sich das nicht grundsätzlich ändert, erweist man den Gewerkschaften einen Bärendienst, wenn man ihnen nahelegt, die Kommission mit einer Kompetenz in Lohnfragen zu versorgen.

\section{Fazit}

Brunkhorsts "Auswege aus der technokratischen Falle " sind Ausdruck einer in der Europadebatte verbreiteten Position, die ich als »integrationistisch « bezeichnet habe. Dieser Position haften mehrere Versäumnisse an: ein Mangel an analytischer Zerlegung des Integrationsprozesses in Dimensionen mit komplexen, einander oft zuwiderlaufenden Beeinflussungsmustern und ein Mangel an politökonomischer Analyse, inklusive einer mangelnden Beachtung der Unterschiedlichkeit der in der EU vertretenen "Spielarten des Kapitalismus « und der Konflikte zwischen ihnen. Problematisch sind diese Versäumnisse, weil sie zu einer systematischen Fehleinschätzung anleiten, die ich als "integrationistischen Fehlschluss « bezeichnet habe. Der Fehlschluss besteht in der Hoffnung, jede weitere Verlagerung von Kompetenzen und politischen Prozessen auf die europäische Ebene werde sich positiv auf das auswirken, wofür gute Europäer letztlich antreten: auf die Entwicklung eines transnationalen "Wir-Gefühls " und die Entstehung der vor-konstitutionellen Voraussetzungen einer europäischen Demokratie im engeren Sinne.

33 Mit Monti II wollte die Kommission die zur Kollision von Grundfreiheiten und Arbeitskampfrecht ergangenen EuGH-Urteile sekundärrechtlich kodifizieren und eine Meldepflicht für Arbeitskämpfe mit transnationalen Auswirkungen durchsetzen. Weil sich eine vertragliche Ermächtigung zur Errichtung eines Überwachungssystems für Streiks nicht behaupten ließ, musste sich die Kommission auf die "Abrundungskompetenz" des Art. 352 AEUV berufen. Diese Kompetenz kann nur unter einstimmiger Zustimmung des Rats genutzt werden. Die "gelben Karten« im Rahmen der Subsidiaritätsrüge signalisierten der Kommission daher, dass Monti II keine Aussichten auf Verwirklichung haben konnte. 
In Bezug auf diese Ziele eines jeden guten Europäers hat der Euro schwere Schäden angerichtet. Er hat uns den Idealen einer europäischen Demokratie nicht nähergebracht, sondern das europäische Demokratiedefizit auf zweierlei Weise radikalisiert: Er hat Konvergenzerfordernisse geschaffen, die sich mit den heterogenen Präferenzen, Praktiken, Institutionen und Prozessen auf Ebene seiner Teilnehmer nicht vereinbaren lassen und denen sich daher nach Ausbruch der Krise nur mit neuen, auf Dauer gestellten und nicht demokratisierbaren supranationalen Befugnissen zur Überwachung und Korrektur mitgliedstaatlicher Politik begegnen ließ. Und er hat eine europäische Konfliktstruktur vertieft, die nicht entlang eines transnational geteilten Gegensatzes zwischen Links und Rechts verläuft, sondern zwischen Ländergruppen, was der Demokratisierbarkeit der Konfliktaustragung seinerseits enge Grenzen setzt und - schlimmer noch - umso mehr an nationalen Ressentiments und Stereotypen erwarten lässt, je mehr sich die Konfliktaustragung politisiert. Solange diese Probleme nicht gelöst sind, werden gut gemeinte Appelle zur politischen Transnationalisierung und Rufe nach mehr Einfluss des EPs im politischen System Europas wenig zur Zielerreichung beitragen. Eine stimmige Analyse der gegenwärtigen Situation, so das Grundanliegen meiner Intervention, sollte verstärkt an der Basis Europas ansetzen: seiner politischen Ökonomie. ${ }^{34}$

\section{Literatur}

Blomert, Reinhard 2012. "Schulden und Schuldige. Deutsche Krisendeutungen «, in WZB-Mitteilungen 137, September 2012, S. 21-24.

Brunkhorst, Hauke 2014 a. "Auswege aus der technokratischen Falle ", in Leviathan 42, 4, S. 507-522.

Brunkhorst, Hauke 2014 b. Das doppelte Gesicht Europas. Frankfurt a. M.: Suhrkamp.

Eppler, Annegret; Scheller, Henrik 2013. "Zug- und Gegenkräfte im europäischen Integrationsprozess", in Zur Konzeptualisierung europäischer Integration. Zug- und Gegenkräfte in europäischen Integrationsprozess, hrsg. v. Eppler, Annegret; Scheller, Henrik, S. 11-42. Baden-Baden: Nomos.

Flassbeck, Heiner; Lapavitsas, Costas 2013. The systemic crisis of the Euro: true causes and effective therapies. Studien der Rosa-Luxemburg-Stiftung. Berlin: Rosa-Luxemburg-Stiftung.

Gerhards, Jürgen; Lengfeld, Holger 2013. Wir, ein europäisches Volk? Sozialintegration Europas und die Idee der Gleichheit aller europäischen Bürger. Wiesbaden: Springer VS.

Hancké, Bob 2013. Unions, central banks, and EMU: labour market institutions and Monetary Union in Europe. Oxford: Oxford University Press.

Höpner, Martin 2014. Wie der Europäische Gerichtshof und die Kommission Liberalisierung durchsetzen: Befunde aus der MPIfG-Forschungsgruppe zur Politischen Ökonomie der europäischen Integration. MPIfG Discussion Paper 2014/8. Köln: Max-Planck-Institut für Gesellschaftsforschung.

Höpner, Martin; Lutter, Mark 2014. One currency and many modes of wage formation. Why the Eurozone is too heterogeneous for the Euro. MPIfG Discussion Paper 2014/14. Köln: MaxPlanck-Institut für Gesellschaftsforschung.

Höpner, Martin; Schäfer, Armin 2015. "Integration among unequals. How the heterogeneity of European varieties of capitalism shapes the social and democratic potential of the EU ", in Handbook of European politics, hrsg. v. Magone, José M., S. 726-746. London: Routledge.

34 Frei nach Jürgen Kaube in der Frankfurter Allgemeinen Zeitung vom 15. Februar 2012, N4. 
Kitschelt, Herbert 1988. "Left-libertarian parties: explaining innovation in competitive party systems «, in World Politics 40, 2, S. 194-234.

Maatsch, Aleksandra 2014. "Are we all austerians now? An analysis of national parliamentary parties' positioning on anti-crisis measures in the Eurozone ", in Journal of European Public Policy 21, 1, S. 96-115.

Pierson, Paul 1996. »The new politics of the welfare state «, in World Politics 48, 2, S. 143-179.

Risse, Thomas 2014. "No demos? Identities and public spheres in the Euro crisis «, in Journal of Common Market Studies 52, 6, S. 1207-1215.

Scharpf, Fritz W. 1999. Regieren in Europa: Effektiv und demokratisch? Frankfurt a. M., New York: Campus.

Scharpf, Fritz W. 2011. "Monetary Union, fiscal crisis and the preemption of democracy ", in Zeitschrift für Staats- und Europawissenschaften 9, 2, S. 194-219.

Scharpf, Fritz W. 2015. "Political legitimacy in a non-optimal currency area ", in Democratic politics in a European Union under stress, hrsg. v. Cramme, Olaf; Hobolt, Sara B., S. 19-47. Oxford: Oxford University Press.

Schulten, Thorsten 2014. Mindestlohnregime in Europa ... Und was Deutschland von ibnen lernen kann. FES-Studie. Berlin: Friedrich-Ebert-Stiftung.

Schulten, Thorsten; Müller, Torsten 2013. „Ein neuer europäischer Interventionismus? Die Auswirkungen des neuen Systems der europäischen Economic Governance auf Löhne und Tarifpolitik ", in Wirtschaft und Gesellschaft 39, 3, S. 291-321.

Streeck, Wolfgang; Elsässer, Lea 2014. Monetary disunion. The domestic politics of Euroland. MPIfG Discussion Paper 2014/17. Köln: Max-Planck-Institut für Gesellschaftsforschung.

Watkins, Susan 2014. »The political state of the Union «, in New Left Review 90, NovemberDecember, S. 5-25. 
Zusammenfassung: Dieser Beitrag antwortet auf Positionen, die Auswege aus der technokratischen Falle der EU in einem Transfer neuer Kompetenzen an die europäische Ebene sowie in einer Stärkung des Europäischen Parlaments sehen, von der sie eine Demokratisierung des politischen Systems der EU erhoffen. Hierbei, so das Argument des Beitrags, handelt es sich um einen integrationistischen Fehlschluss. Die Notwendigkeit des technokratischen Regierens ist vielmehr im Euro angelegt. Diese Notwendigkeit würde durch ein stärkeres EP nicht verschwinden, und eine stärkere Politisierung europäischer Konflikte könnte die EU von den vor-konstitutionellen Voraussetzungen demokratischer europäischer Prozesse sogar weiter entfernen. Dem integrationistischen Fehlschluss liegen drei Analysefehler zugrunde: zu wenig analytische Zerlegung des Integrationsprozesses in Dimensionen der Integration, zu wenig politökonomische Fundierung und eine Unterschätzung der europäischen Konfliktlinien, die nicht zwischen Links und Rechts, sondern zwischen Ländern verlaufen.

Stichworte: europäische Integration, politisches System der EU, Demokratie, Politische Ökonomie, Währungsunion

\section{The integrationist fallacy}

Summary: This contribution is a response to those who argue that more EU competencies and a stronger European Parliament would free Europe from the technocratic trap and establish a European-wide democracy. The respective position relies on an integrationist fallacy. The necessity for technocratic governance is built into the monetary union and would therefore not vanish if the EP became stronger. Also, more politicization of European conflicts may drive the EU further apart from the pre-constitutional conditions of transnational democratic processes. The integrationist fallacy relies on three shortcomings: the lack of analytical differentiation between integration dimensions, the absence of a political economic perspective, and too little attention on conflicts between countries as opposed to those between the left and the right.

Keywords: European integration, political system of the EU, democracy, political economy, monetary union

Autor

apl. Prof. Dr. Martin Höpner

Max-Planck-Institut für Gesellschaftsforschung

Paulstraße 3

50676 Köln

hoepner@mpifg.de 